\title{
A MICROMACHINED INTERFACE FOR TRANSFER OF LIQUID OR VAPOUR SAMPLE TO A LIQUID SOLUTION
}

\author{
Wouter van der Wijngaart, Thomas Frisk, Göran Stemme \\ Microsystem Technology, Dept. of S3, Royal Institute of Technology, Stockholm, Sweden \\ e-mail: wouter@s3,kth,se
}

\begin{abstract}
A novel microfluidic interface for vapour-to-liquid adsorption and droplet-to-liquid transfer was designed and fabricated using silicon micromachining. The interface was characterised and successfully tested.
\end{abstract}

Keywords: liquid interfacing, microfluidics, $\mu$ TAS

\section{INTRODUCTION}

Sample-to-liquid interfacing is required in all $\mu$ TAS (micro total analysis systems) systems where chemical analysis of dust or vapour samples is performed in liquid environment. Interfacing micro droplets to liquid was shown previously [1]. The device presented here provides an open liquid-air interface for direct vapourto-liquid (alternatively liquid-to-liquid) sample transfer.

Component design requirements include:

1. Providing large interface area $\left(\sim \mathrm{cm}^{2}\right)$ to a small liquid volume to obtain high sample concentration.

2. Preventing liquid buffer salinity changes by preventing liquid evaporation.

3. Easy device liquid priming, e.g. by capillary filling.

4. Providing robustness in terms of preventing device flooding.

5. Providing robustness in terms of preventing drying or downstream air bubble introduction.

6. Allowing sufficient liquid flow $(\sim \mu \mathrm{l} / \mathrm{s})$.

7. Low flow resistance $(\sim \mathrm{kPa})$.

For "in the field" use demands include:

8. Gravitational invariance.

9. Easy cleaning and regeneration of the device.

\section{DEVICE FUNCTIONALITY}

The interface consists of a micromachined surface over which a constant liquid flow is guided in contact with the surrounding atmosphere (Fig. 1). The device surface consists of a hexagonal pattern of cylindrical pillars on a silicon substrate.

Liquid flows on the chip surface between the pillars, with surface tension preventing liquid to wet the pillar tops.
Liquid is added and removed from the silicon chip via Teflon ${ }^{\mathrm{TM}}$ or PDMS fluid connectors with stainless steel tube interfaces. The internal geometry of the connectors is shown in Fig. 1. They feature a manifold design for even filling and draining of the device. The silicon surface is primed capillarily.

The liquid surface forms a condensation site for vapour or aerosol sample. Condensation occurs preferably when the interface liquid is cooled, the latter also reducing liquid evaporation.

The capillary forces between the pillars prevent drying and air bubbles entering downstream the interface outlet.

Hydrophobicity and hydrophilicity of the component's surfaces has a huge impact on the performance of the device. Care must be taken of in material choices and device geometry. The fluid connectors being hydrophobic prevents overflow at the connector-chip-air contact line.

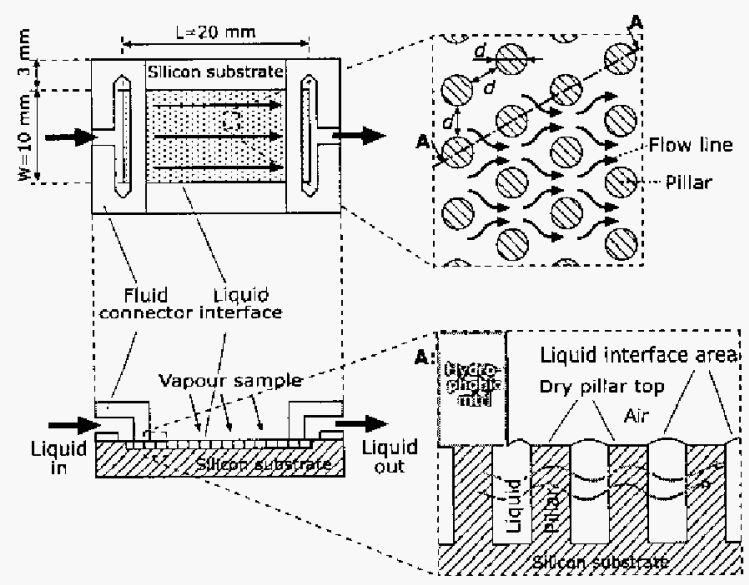

Fig. 1. Schematic top view (top) and cross-sectional view (bottom) of the liquid guiding surface geometry.

Vapour, liquid or particles can adsorb to the liquid interface. When a flow is maintained, the sample is carried through the device for downstream analysis.

\section{FABRICATION}

The silicon surface microstructuring was performed with standard methods including a single-mask design, photo-lithography, DRIE and final dicing.

\section{TRANSDUCERS'05}

The 13 th International Conference on Solid-Staze Sensors, Actuators and Microsystems, Seoul, Korea, Jume 5-9, 2005 
The active area width $W(=1 \mathrm{~cm})$, length $L(=2 \mathrm{~cm})$, pillar diameter $d(=20-40 \mu \mathrm{m})$, height $h(=50-100 \mu \mathrm{m})$ and inter-pillar distance $d$ provides a $\sim 2 \mathrm{~cm}^{2}$ interface area for a $10-20 \mu \mathrm{l}$ liquid volume.

The silicon chips were clamped between the fluid connectors (Teflon ${ }^{\text {TM }}$ or PDMS) and PMMA support using screws.

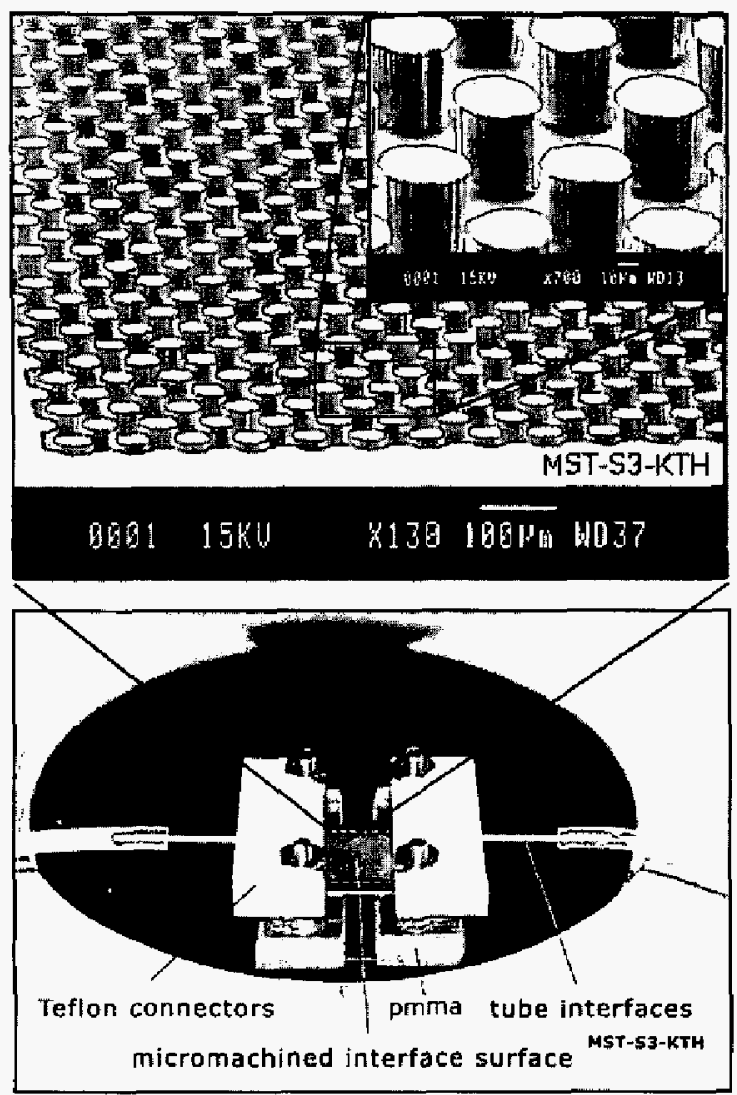

Figure 2. Photograph of a $2 \times 1 \mathrm{~cm}^{2}$ interface chip with Teflon ${ }^{\mathrm{TM}}$ connectors and stainless steel tube interfaces with silicone tubing (bottom). SEM image showing the microstructured silicon pillar surface with etch depth $50 \mu \mathrm{m}$ (top).

\section{DESIGN PARAMETERS}

The flow behaviour of liquid on a regularly microstructured open surface is described as a 2-D extension of flow through a single open microcanal [2].

In our theoretical analysis we model the device surface as $n=W / d$ parallel microcanals of width $d$ and height $h$ and we assume parallel plate Poiseuille flow (i.e. we neglect the channel bottom).
The viscous flow resistance (pressure drop per flow) for such geometry is

$$
\frac{\Delta P_{i}}{Q}=\frac{48 L \mu}{W h d^{2}}
$$

Note that the internal liquid pressure drops along the length $L$ of the liquid interface, and that the liquid pressure at the upstream side of the device is always larger than at the downstream side.

Two device failure mechanisms exist, shown in Fig. 3

Device flooding (i.e. liquid covering the pillar tops) occurs where the internal liquid pressure

$$
P_{l}>P_{f l}=\frac{2 \gamma}{d} \sin \theta_{c} .
$$

Device pinch-off (i.e drying) and downstream air bubble introduction occur where

$$
P_{i}<P_{p o}=-\frac{\gamma}{d} \cos \theta_{c}
$$

The spacing $d$ between the pillars forms a critical design parameter, and its choice is a trade-off.

On one hand, eq. 1 shows that the viscous pressure drop increases quadratically with decreasing interpillar spacing. Depending on whether the flow is controlled with a pressure source (c.g. hydrostatically) or with a flow souree (e.g. syringe pump), and depending on whether the flow control is upstream or downstream of the device, a large pressure drop over the device can lead to either flooding on the upstream side, or pinch-off at the downstream side.

On the other hand, eq. 2 and 3 show that decreasing inter-pillar spacing decreases the pinch-off pressure (there is relatively more wetting surface available, i.e. more capillary action) and increases the overflow robustness. However, these effects scale only linear with $d$.

The choice of pillar height $h$ is also a trade-off. On one hand, small values of $h$ increase the interface area per liquid volume, and thus the sample concentration in the adsorbing liquid. The decreased flow crosssectional area, on the other hand, increases the viscous pressure drop over the device, and thus the risk for flooding or pinch-off. 


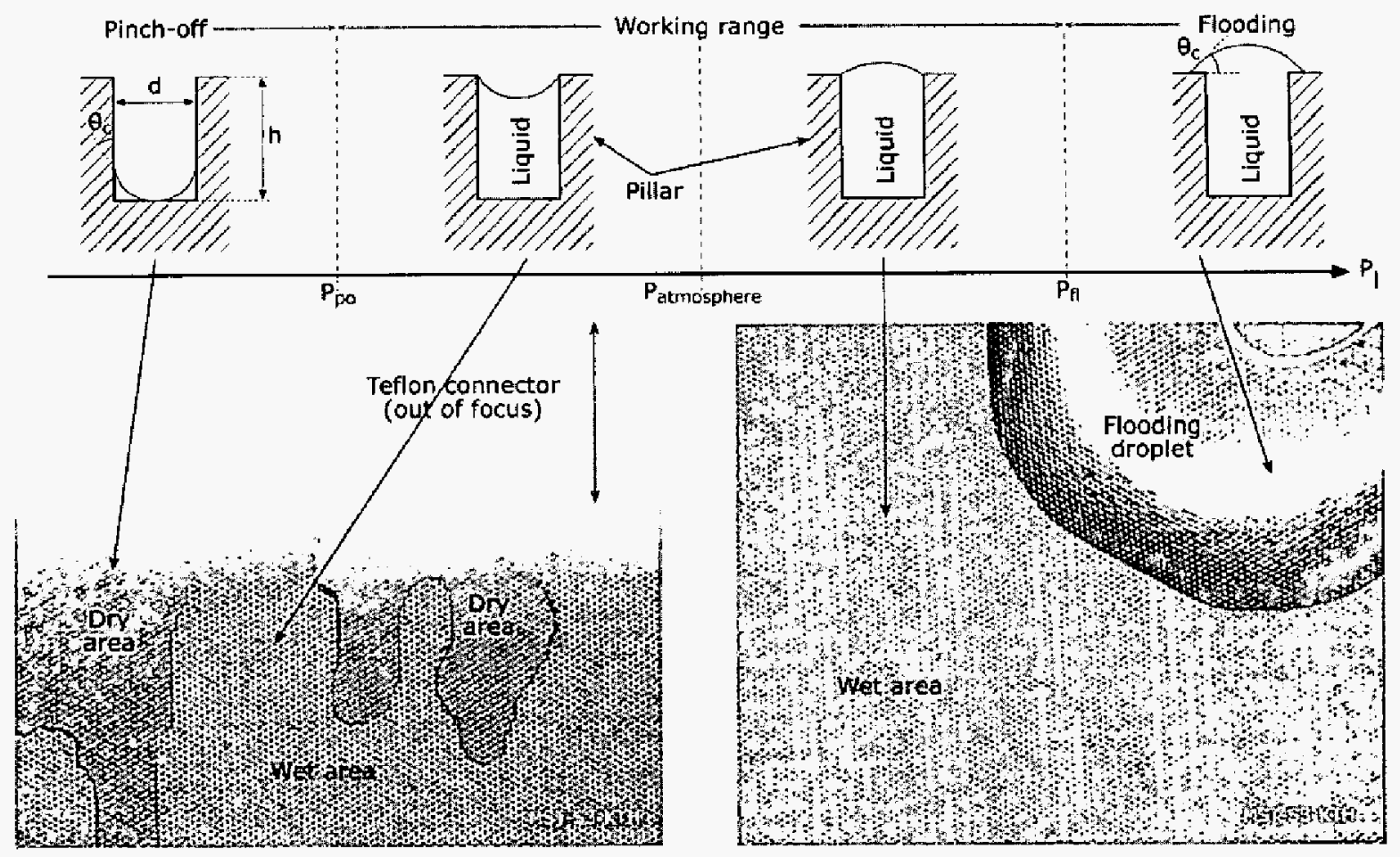

Fig. 3. Schematic cross-sectional view of wetting and liquid-air interface geometry at different internal liquid pressures $P_{l}$ (top) and microscope photographs illustrating the different states (bottom).

\section{EXPERIMENTAL}

The pressure-flow measurement setup with hydrostatic upstream and downstream pressure control is shown in figure 4 . The device was positioned at a fixed height. Inlet and outlet pressure were controlled hydrostatically, while the mass flow was measured with a scale at the outlet. Measurement results are shown in figure 6. Pinch-off and overflow regimes (Fig. 3) were investigated.

The system was also successfully tested for liquid droplet interfacing. Drops of dye were deposited on the interface surface, and the migration of the coloured liquid studied for flow pattern and residing time/exchange rate in device. The liquid residence time in the interface varied typically between 3 and $10 \mathrm{~s}$ depending on the flow rate.

For in-the-field robustness the gravity invariance was tested through varying the position/orientation of the device. We tested the device turned up side down as well as tilted and tilted-turned (Fig. 7). With device up side down (B) we observed no disturbed functionality.
With the device vertical (C\&D) we investigated priming as well as pinch-off, finding only slight performance difference compared to the horizontal position (A). Neither in the tilted position (E) was any significant change in performance detected.

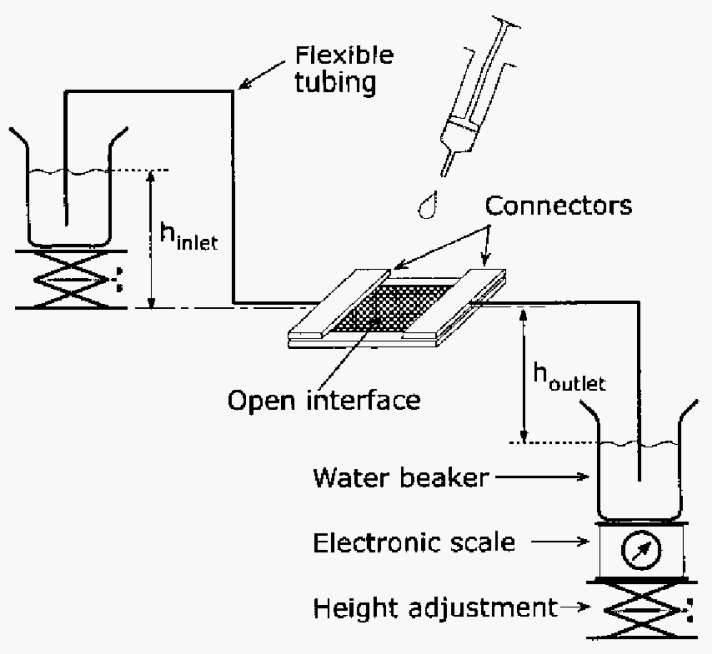

Fig. 4. Schematic drawing of the experimental setup. 


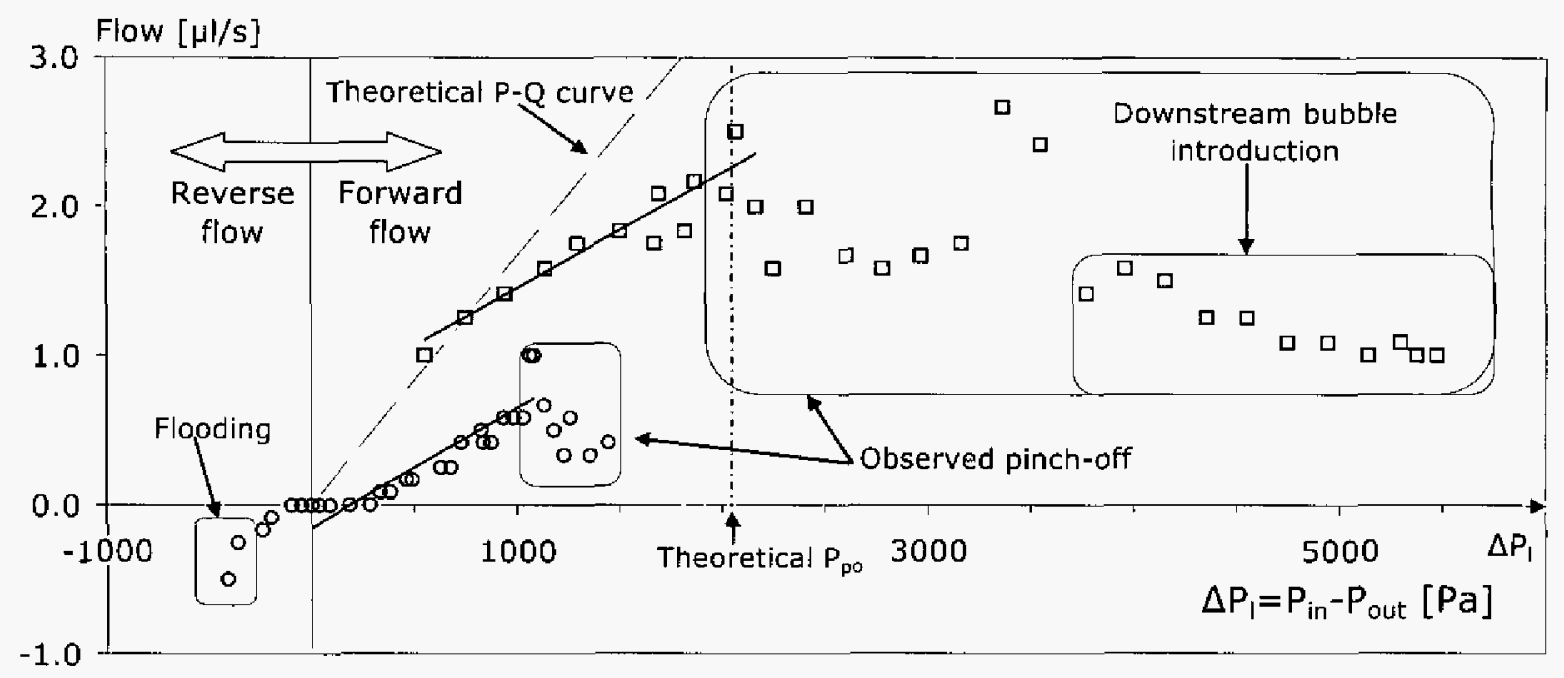

Fig. 5. Pressure-flow measurements with $d=40 \mu \mathrm{m}$ and $h=100 \mu \mathrm{m}$. Viscous losses in the connectors are negligible. The inlet pressure was set at $+175 \mathrm{~Pa}$ (squares), respectively $-250 \mathrm{~Pa}$ (circles) while the outlet pressure was varied. In the latter measurement series, $P_{K}<P_{a t m}$ at all time. The dashed line indicates the model predictions (eq. 1); solid lines indicate linear curve fits to the data in the working range.

Liquid antibody sample and narcotic vapour sample interfacing was successfully performed [3] in conjunction with a commercial Biosens ${ }^{\mathrm{TM}}$ electronic nose system [4].
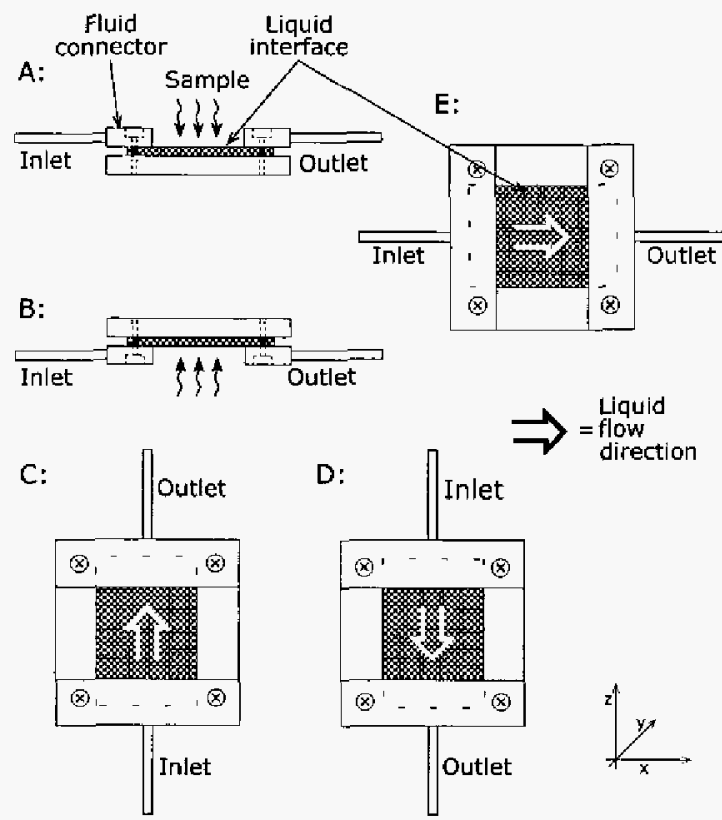

Fig. 6. Illustrations of the different device positions tested to investigate the gravitational invariance of the device. A show the orientation of the device for which the P-Q characterisation measurements were carried out. In positions $B$ to $E$ we tested priming and filling as well as pinch-off and dry-out, not finding any significant changes in performance.

\section{CONCLUSION}

A novel uncomplicated microfluidic interface for vapour-to-liquid adsorption and droplet-to-liquid transfer was designed and fabricated.

Its characteristics fall within the commercially required range, and can easily be modified to accomplish different demands.

\section{ACKNOWLEDGEMENTS}

This work was funded by VINNOVA through the SUMMIT program. We would like to thank Kjell Norén at the MST Laboratory at KTH for the help with manufacturing of the fluid connectors.

\section{REFERENCES}

[1] GeSiM mbH, Germany, URL: www.gesim.de.

[2] Melin et al, "Behaviour and Design Considerations for Continuous Flow ClosedOpen-Closed Liquid Microchannels", Lab on a Chip, accepted for publication, 2005.

[3] Frisk et al, "Fast narcotics and explosives detection using a microfluidic sample interface", Transducers 05 Technical Digest.

[4] Biosensor Applications AB, Sweden, URL: www.biosensor.se. 